\title{
ANGIOMIOLIPOMA RENAL GIGANTE
}

\author{
G. GUTIÉRREZ FERNÁNDEZ, A. MANSILLA ROSELLÓ, F. RUBIO GIL, \\ A.P. MARTÍNEZ DOMÍNGUEZ, J. VILLAR DEL MORAL, A. FERRÓN ORIHUELA \\ Departamento de Cirugía General y del Aparato Digestivo. Servicio de Cirugía General y del Aparato \\ Digestivo. Hospital Médico Quirúrgico. Hospital Universitario Virgen de las Nieves. Granada.
}

Actas Urol Esp. 27 (6): 462-464, 2003

\section{RESUMEN}

ANGIOMIOLIPOMA RENAL GIGANTE

Presentamos un caso de angiomiolipoma renal cuya peculiaridad es el gran tamaño que tiene en el momento del diagnostico lo que ocasiona una alteración de la morfología del riñón afecto, así como al resto de vísceras de la cavidad abdominal. Por este motivo se interviene realizando exéresis de la masa con nefrectomía. Realizamos una revisión bibliográfica de este tumor analizando sus peculiaridades y aspectos más destacables.

PALABRAS CLAVE: Angiomiolipoma. Tumor renal. Tumor retroperitoneal.

\section{ABSTRACT}

\section{GIANT RENAL ANGIOMYOLIPOMA}

We present a case report of a renal angiomyolipoma with the special feature of its big size at the moment of the diagnosis. It is appreciated an important alteration of the kidney morphology and the repercussion produced in the rest of the abdominal organs. Due to this an exeresis with nefrectomy is performed. We do a bibliographic review and we analyzed the relevant aspects of this tumour.

KEY WORDS: Angiomyolipoma. Renal tumour. Retroperitoneal tumour.

L os angiomiolipomas renales son tumores benignos poco frecuentes. Tienen dos modalidades de presentación según el contexto en que se presenten. La actitud frente a esta lesión suele ser conservadora aunque deben analizarse las peculiaridades de cada caso. Presentamos un caso en el que la extirpación quirúrgica del tumor fue necesaria debido a las grandes dimensiones que había adquirido a lo largo de varios años de evolución.

\section{CASO CLÍNICO}

Mujer de 26 años de edad que es remitida desde el Servicio de Oncología Médica para estudio de masa abdominal retroperitoneal. No presenta ningún antecedente patológico de interés. Ella refiere que en los últimos siete años nota cre- cimiento progresivo del perímetro abdominal sin que ello le produzca ninguna sintomatología específica.

A la exploración presenta buen estado general, destaca la exploración abdominal donde se palpa una gran masa dura de consistencia elástica que abarca todos los cuadrantes abdominales excepto ambas fosas iliacas.

Analíticamente todos los parámetros tanto bioquímicos como hematológicos están dentro de la normalidad. Creatinina de $0,8 \mathrm{mg} / \mathrm{dl}$ y urea 50 $\mathrm{mg} / \mathrm{dl}$. Marcadores tumorales sin alteraciones.

En la tomografía computerizada (TC), se aprecia gran masa retroperitoneal, que parece depender del riñón derecho (Fig. 1), de densidad grasa, bordes bien delimitados y vasos engrosados. Produce compresión y atrofia del riñón, con cierta 


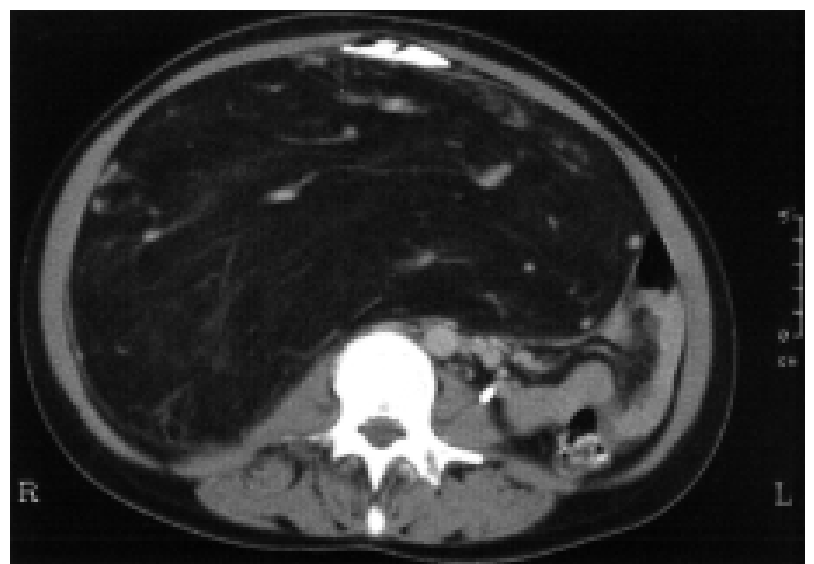

FIGURA 1: TAC abdominal con la masa retroperitoneal.

hidronefrosis. Se sugiere el diagnóstico de angiomiolipoma sin embargo debido a su gran tamaño se establece el diagnóstico diferencial con un liposarcoma de la grasa perirrenal.

Con el diagnóstico de tumor retroperitoneal gigante se decide intervención quirúrgica evidenciando en el acto operatorio una masa que depende del riñón derecho, bien delimitada, de $40 \times 30 \times 12 \mathrm{~cm}$, al que comprime junto con la glándula suprarrenal (Fig. 2), también inmersa en el tumor. Es destacable la presencia de grandes vasos aberrantes que, junto con el gran tamaño dificultan la cirugía (Fig. 3). Se realiza exéresis de la tumoración con nefrectomía (Fig. 4). La paciente es dada de alta a los pocos días siendo su evolución postoperatoria favorable.

El informe anatomopatológico informa de: angiomiolipoma renal con vasos aberrantes.

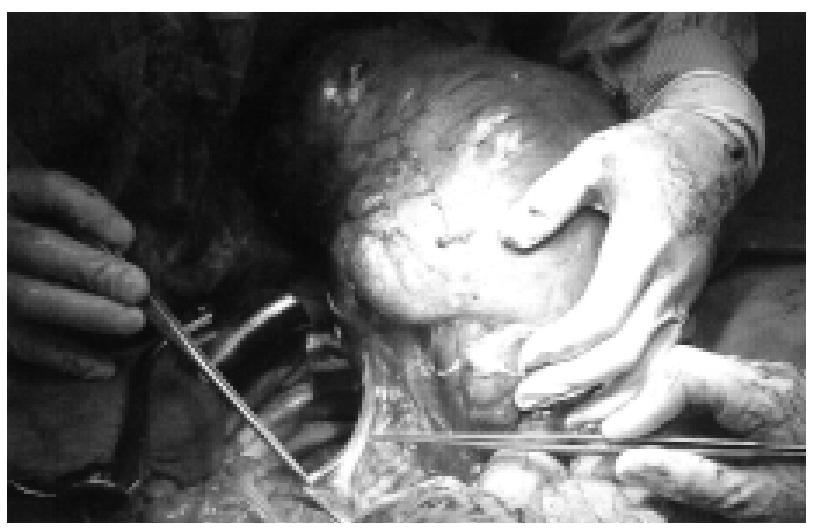

FIGURA 2. Imagen del tumor con el pediculo renal.

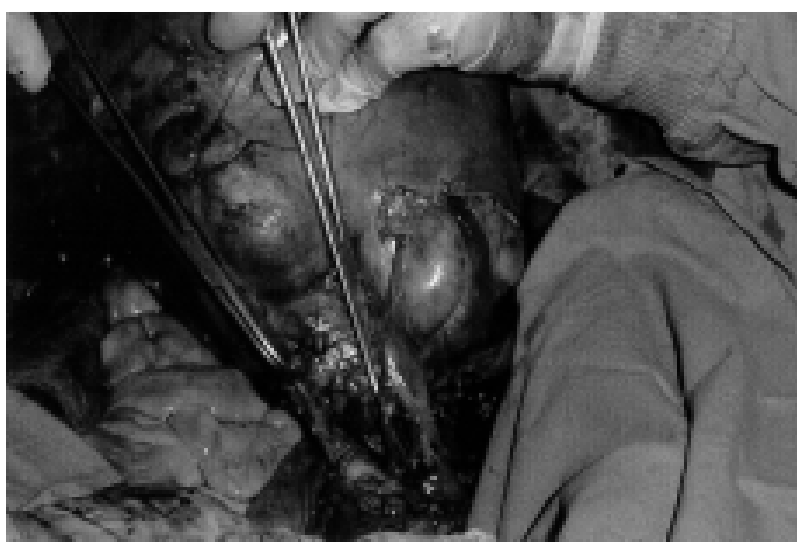

FIGURA 3. Imagen de los vasos aberrantes.

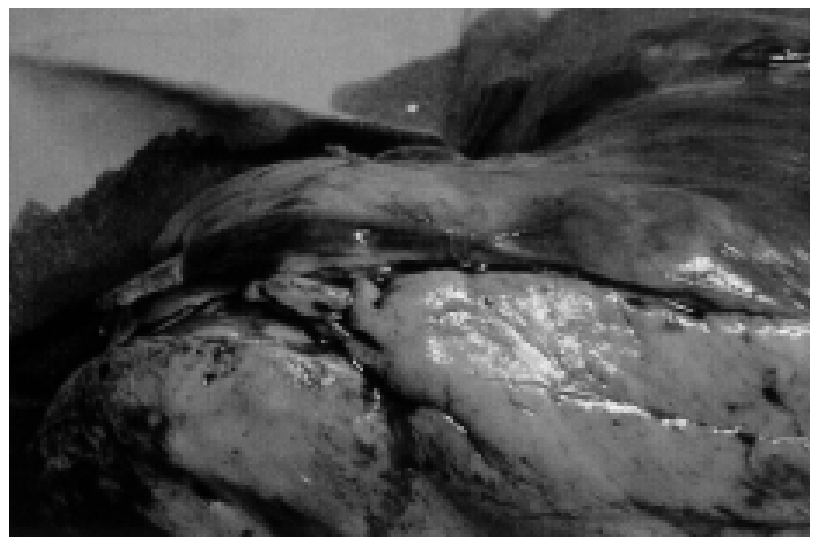

FIGURA 4. Imagen del riñón, comprimido por la masa subyacente.

\section{DISCUSION}

Los angiomiolipomas renales son tumores benignos poco frecuentes. En la práctica clínica tienen dos formas presentación. Una modalidad en la que asocian a enfermedades hereditarias, (facomatosis), de las que la esclerosis tuberosa es la más importante. En esta se asocia en un $80 \%^{1,2}$ de los casos, presentándose junto con la triada clásica de retraso mental, adenomas sebáceos y epilepsia. Suele afectar a mujeres siendo tumores bilaterales, pequeños y por lo general asintomáticos ${ }^{3}$. De forma más extraordinaria se pueden encontrar en otras facomatosis como la neurofibromatosis de von Recklinhausen o la enfermedad de Von Hippel Lindau ${ }^{3}$. La otra forma de presentación es aislada. Aquí suelen ser unilaterales, también por lo general asintomáticos, con tendencia a ser pequeños aunque presentan mayor variabilidad en el tamaño, teniendo una incidencia mayor en la mujer con relación $3: 1^{3}$. 
Independientemente de la presentación que tengan, asociado o no a la esclerosis tuberosa, histológicamente son iguales. Macroscópicamente están compuestos de grasa, músculo liso y vasos anormales en diferente proporción, son tumores mayoritariamente amarillentos de consistencia firme auque será la concentración predominante de grasa o músculo la que determina su apariencia externa. En nuestro caso el gran tumor era amarillento con grandes vasos que recorrían su superficie.

Si bien clínicamente son asintomáticos pueden producir diferente sintomatología. Puede haber simple sensación de masa en flanco, dolor abdominal o dispepsia, hematuria, o presentarse como un abdomen agudo por hemorragia desde el tumor, necrosis o rotura ${ }^{1,3}$. Sorprende que nuestra paciente a pesar del gran tumor no refiriera dolor abdominal sino sensación de masa y dispepsia.

Con el uso habitual de la ecografía en exploraciones abdominales la detección como hallazgo casual se ha incrementado ${ }^{3}$. Gracias a su composición lipídica poseen una ecogeneicidad característica por lo que son fácilmente detectables así como por T.C. ${ }^{2}$. Sin embargo, ocasionalmente, puede haber dudas diagnosticas respecto a la estirpe tumoral por lo que, en estos casos, la exploración quirúrgica esta justificada, ya que por esos vasos aberrantes la punción para biopsiar podría producir hemorragias tumorales ${ }^{3}$.

Dentro del tratamiento quirúrgico hay variedad de actuaciones. Se han descrito embolizaciones previas a la cirugía como terapia inicial para prevenir la posible hemorragia favorecida por la presencia de esos vasos atípicos, resección del tumor con nefrectomías parciales o totales, según las características del tumor (tamaño, relaciones anatómicas etc. $)^{5}$. El tumor que a nosotros se nos presentó tenia un tamaño tan grande y afectaba de una manera tan importante al riñón y glándula suprarrenal que no se puedo evitar la nefrectomía al realizar la exéresis del tumor.

Un punto de polémica ha sido el tratamiento más adecuado para este tumor benigno. Según diferentes series ${ }^{4-6}$, tumores menores de $4 \mathrm{~cm}$. suelen permanecer asintomáticos en el tiempo. Tumores mayores de $4 \mathrm{~cm}$ más frecuentemente (52\%) son sintomáticos y la cirugía estaría indicada en algún caso ${ }^{4}$. Los tumores pequeños crecen muy despacio y lo hacen de manera inconstante $^{3}$. Revisiones periódicas anuales o bianuales han sido sugeridas, y solo el caso de que aparezcan síntomas o se detecte crecimiento o haya dudas diagnósticas, la intervención quirúrgica estaría indicada.

\section{REFERENCIAS}

1. WONG AL, MCGEORGE A, CLARK AH.: Renal angiomyolipoma: a review of the literature and a report of 4 cases. Br J Urol 1981; 53 (5): 406-411.

2. MALONE MJ, JOHNSON PR, JUMPER BM, HOWARD PJ, HOPKINS TB, LIBERTINO JA.: Renal angiomyolipoma: 6 cases reports and literature review. J Urol 1986; 135 (2): 349-353.

3. DE LUCA S, TERRONE C, ROSSETTI SR.: Management of renal angiomyolipoma: a report of 53 cases. BJU Int 1999; 83 (3): 215-218.

4. STEINER MS, GOLDMAN SM, FISHMAN EK, MARSHALL FF.: The natural history of renal angiomyolipoma. J Urol 1993; 150 (6): 1782-1787.

5. DICKINSON M, RUCKLE H, BEAGHLER M, HADLEY HR.: Renal angiomyolipoma: optimal treatment based on size and symptoms. Clin Nephrol 1998; 49 (5): 281-286.

6. LINGEMAN JE, DONOHUE JP, MADURA JA, SELKE F.: ANGIOMYOLIPOMA: Emerging concepts in management. Urology 1982; 20 (6): 566-570.

Dr. G. Gutiérrez Fernández

C/ Lance, s/n. Edif. Emperatriz - Portal 4, 5ㅇ A 18014 Granada

(Trabajo recibido el 16 julio de 2002) 